\title{
The effect of Soya milk on the total antioxidant capacity of black tea
}

\author{
L. Ryan and S. Sutherland \\ Functional Food Centre, School of Life Sciences, Oxford Brookes University, Gipsy Lane, Headington, \\ Oxford OX3 OBP, UK
}

Tea (Camellia sinensis), one of the most commonly consumed beverages in the world, is a rich source of polyphenolic compunds. To date, a range of epidemiological evidence has suggested that the consumption of polyphenols may be associated with a decreased risk of CHD, stroke incidence, chronic inflammation and cancer incidence. As black tea is widely consumed in the UK (3-4 cups of tea per day compared to 1-2 cups of tea per day for other countries), the antioxidant components of black tea may contribute significantly to the overall antioxidant intake in the UK diet. However, it is common practice in the UK to add milk to tea and some studies have suggested that this may decrease the bioavailability and the antioxidant potential of polyphenols in vitro and in vivo ${ }^{(1)}$. There are limited data on the effects of soya milk on the potential availability of black tea antioxidants. The objective of the present study was to analyse and compare the antioxidant capacity of four brands of tea and to investigate the effect of the addition of different varieties of soya milk on the total antioxidant capacity of the teas.

Four brands of tea were chosen (Typhoo, Tetley, Twinnings Everyday and PG Tips). In all experiments, tea preparations were made using a standard protocol. Each tea was infused in $200 \mathrm{ml}$ of boiling water $\left(90^{\circ} \mathrm{C}\right)$. Teas were analysed individually and after the addition of $20 \mathrm{ml}$ water $\left(\mathrm{H}_{2} \mathrm{O}\right)$, skimmed milk (SK), and different types of soya milk; Alpro Original (AO), Alpro Unsweetened (AU), Alpro Light (AL), Provamel (Pro) and Holland \& Barrett (HB). The total antioxidant capacity of the samples was determined using the FRAP assay ${ }^{(2)}$.

\begin{tabular}{|c|c|c|c|c|c|c|c|c|c|c|c|c|c|c|}
\hline & \multicolumn{14}{|c|}{ FRAP $(\mu \mathrm{mol} / \mathrm{l})$} \\
\hline & $\mathrm{H}_{2} \mathrm{O}$ & SE & SK & $\mathrm{SE}$ & $\mathrm{AO}$ & SE & $\mathrm{AU}$ & $\mathrm{SE}$ & $\mathrm{AL}$ & $\mathrm{SE}$ & Pro & $\mathrm{SE}$ & HB & SE \\
\hline Typhoo & 6045 & 63 & $4997^{*}$ & 46 & 5819 & 211 & 5393 & 159 & 6232 & 59 & 6134 & 158 & 5894 & 36 \\
\hline Tetley & 6191 & 93 & $5116^{*}$ & 90 & 5896 & 157 & 5730 & 160 & 5893 & 131 & 5875 & 36 & 6159 & 175 \\
\hline Everyday & 6354 & 8 & $5386^{*}$ & 36 & 6575 & 43 & 5935 & 180 & 6247 & 91 & 5748 & 183 & 6287 & 266 \\
\hline PG Tips & 7021 & 8 & $5907^{*}$ & 4 & $5918 *$ & 39 & 6769 & 39 & 6920 & 263 & 6904 & 137 & 6933 & 41 \\
\hline
\end{tabular}

$n 3$ independent experiments; $* P<0.05=$ significantly different from same tea sample with $20 \mathrm{ml}$ of water added.

Each of the teas analysed was a significant source of antioxidants. For all brands of tea, the addition of $20 \mathrm{ml}$ of SK significantly $(P<0.05)$ decreased the total antioxidant capacity compared to tea with the same volume of water added. None of the soya milks significantly decreased the total antioxidant capacity of Typhoo, Tetley or Everyday teas. PG Tips had the highest antioxidant capacity out of the teas analysed. The addition of Alpro Original soya milk to a PG Tip tea infusion decreased the total antioxidant capacity by $\sim 16 \%$. None of the other soya milks significantly decreased the antioxidant capacity of this tea. In conclusion, black tea is a valuable source of antioxidants and the addition of soya milk to black tea may be a useful alternative to SK if one intends to maintain the total antioxidant capacity of the beverage.

1. Ryan L \& Petit S (2010) The addition of whole, semi-skimmed and skimmed bovine milk reduces the total antioxidant capacity of black tea. Nutr Res 30, 14-20.

2. Benzie IFF \& Szeto YT (1999) Total antioxidant capacity of teas by the ferric reducing antioxidant power assay. J Agric Food Chem 47, $633-636$. 